\title{
Francisolā
}

\section{DÉNOMINATION PARALLÈLE DE RUES EN ALGÉRIE : CONFLIT SUR LES MOTS ET CONFLITS SUR LE SENS}

\author{
Djemaa TIMZOUERT
}

\author{
Université M’Hamed Bougara de Boumerdes, Algérie \\ timzouertd@yahoo.com
}

Article reçu le 15 novembre 2016 | révisé depuis le 2 mars 2017 | accepté le 30 juin 2017

\begin{abstract}
RÉSUMÉ. L'ensemble suivant est une étude praxématique de discours épilinguistiques autour d'odonymes parallèles dans la ville de Tizi-Ouzou, en Algérie. Il est question, d'un côté, d'analyser, au moyen des principes de la linguistique de la parole associés à l'onomastique, du fonctionnement discursif de ces productions langagières tenues au sujet du recours aux dénominations autres que celles consacrées officiellement pour désigner les rues au sein de cette ville, et, de l'autre, de saisir le réglage social et conflictuel du sens de ces mises en discours co-construites en interaction verbale avec les participants à l'enquête sociolinguistique que nous avons menée à ce propos.
\end{abstract}

Mots-clés : discours, odonyme, praxématique, sociolinguistique

\begin{abstract}
The following article is a study of epilinguistiques speech about parallel odonyms in the city of Tizi-Ouzou, Algeria. It is question, on one side, to analyze, through the principles of Linguistics of the significance associated with onomastics, discursive these language production operations held to the subject of the use of names other than those dedicated officially to designate streets within the city, and, on the other, to seize the setting social and confrontational sense of such speech co-created in verbal interaction with participants in the sociolinguistic survey that we conducted to these remarks.
\end{abstract}

Keywords : discourse, odonym, praxematics, sociolinguistics

\section{INTRODUCTION}

En adoptant la théorie de la construction dynamique du sens en langage, la praxématique, nous nous proposons, dans cette étude, d'analyser des discours épilinguistiques tenus autour des désignations parallèles des rues au niveau de la ville de Tizi-Ouzou, en Algérie. La problématique du réglage social du sens que ses mises en discours infèrent ou qui serait à la base de leur co-construction est l'un des volets traités dans cette contribution. Il est question de faire ressortir les enjeux complexes de la coproduction des mises en discours recueillies, c'est-à-dire analyser, d'un point de vue praxématique, le "conflit sur les mots et [les] conflits sur le sens » (Barbéris, 2007b, p. 77) de ces productions langagières qui fonctionnent plutôt comme des condensés de sens complexes. Ce qui fait l'objet de ce travail, ce sont moins les représentations autour de ces attributs parallèles de rues dans cette ville algérienne, comme produits définitifs, que les processus linguistique, dialogique et dialogal de formation de celles-ci. La problématique à la 
base de cette étude permet, par ailleurs, de formuler des interrogations au sujet du conflit caractérisant la dénomination des rues de même que la ré-appropriation de ces espaces. Elle met en perspective l'état de carence dénominative officielle des voies urbaines dans la même ville.

Sur le plan social, étant donné $\mathrm{qu}^{\prime}$ «il est important que l'espace soit structuré, [en matière odonymique], de manière rigoureuse et réglementaire selon des critères universels » (Timzouert, 2015, p. 8), cette contribution soulève des questionnements au sujet des déficiences caractérisant le domaine onomastique au sein de la ville de TiziOuzou. L'étude met également en avant la nécessité d'œuvrer en faveur d'une politique onomastique adaptée et qui tient compte des carences dénominatives soulevées autant à Tizi-Ouzou qu'au niveau d'autres villes algériennes. En effet, à l'aire de l'évolution dans divers sens des espaces d'habitation et différentes infrastructures aussi bien dans la ville de Tizi-Ouzou, que dans d'autres villes algériennes, il nous paraît nécessaire, pour les institutions étatiques chargées de nommer les rues et autres places publics, de mettre en œuvre une politique onomastique conforme et appropriée. Car, «la dénomination et sa gestion relève désormais du fonctionnement moderne de la société " (Atoui et Benramdane, 2005, p.187).

Le recourt à des appellatifs autres que ceux adoptés officiellement pour désigner parallèlement les rues (et autres espaces publics) à Tizi-Ouzou, ou ailleurs en Algérie, a des retombées sociales à ne pas négliger mais à considérer. Effectivement, l'usage de ces désignations parallèles, du moins pour les interlocuteurs ayant pris part à l'enquête sociolinguistique menée pour la construction $\mathrm{du}$ corpus à la base de cette étude, a un impact tant sur les domaines d'activité divers, comme celui de la sécurité, les postes, etc., que sur le secteur ayant trait à la circulation routière en milieu urbain, à cause $\mathrm{du}$ manque d'éléments d'indication et d'orientation.

\section{MÉTHODE}

Le travail vise, d'une part, la saisie du fonctionnement discursif des productions langagières co-cosntruites dans un lien d'intersubjectivité avec les partenaires de l'échange ayant pris part à l'enquête sociolinguistique que nous avons menée à la fin de l'année 2013 et au début de l'année 2014 au sujet du recours à des dénominations parallèles, autres que celles adoptées par les instances chargées de la nomination des rues et autres espaces publics en Algérie. Pour mener à bien l'étude, nous postulons l'hypothèse méthodologique selon laquelle les discours épilinguistiques tenus au sujet des dénominations parallèles de rues au sein de la ville de Tizi-Ouzou, en Algérie, ont un soubassement social se manifestant par des praxèmes qui renvoient à des référents sémantiques complexes.

La construction du corpus à base des mises en mots provoquées et coproduites dans l'interaction verbale et intersubjective avec les participants à l'enquête sociolinguistique que nous avons menée revêt un aspect dynamique et conflictuel imposant, d'ailleurs, l'adoption de la linguistique praxématique comme approche à la fois du recueil et d'étude des paroles élaborées en discours oral. Inspirée des réflexions linguistiques de Guillaume (1998) autour de la psychomécanique du langage, c'est-à-dire le lien entre la langue et la pensée, cette théorie a établi au fur et à mesure ses fondements au cours d'un séminaire d'analyse textuelle animé par Robert Lafont au début des années 1970. Au carrefour de la sociolinguistique, de la psychanalyse, de la sémiotique et de la sociologie, Lafont et Madray (1976) développent une vision dynamique des rôles dans l'interaction langagière, des représentations et des conflits. «Théorie de la production linguistique $\mathrm{du}$ sens qui a explicitement posé les principes à partir desquels elle s'applique à élaborer des modalisations de la signifiance en langage» (Siblot, 1988, p. 73), cette approche «dépasse la dichotomie saussurienne langue/parole dans laquelle s'ancrent les développements structuralistes [...] d'une conception qui traite le sens en produit et omet la question 
du comment ce sens est-il produit? Selon quelle dynamique discursive?» (Barbéris et Madray, 1986, p. 37). C'est surtout dans Le travail et la langue, paru en 1978, qu'ont été synthétisées par R. Lafont les propositions principales de cette théorie de la construction dynamique du sens. La recherche dans ce domaine linguistique s'est depuis poursuivie en un cercle de praxématiciens qui regroupe, entre autres, P. Siblot (1986, 1988, etc.), J. Bres $(2006, . .)$, F. Madray $(1984,1989, \ldots)$, et J.-M. Barbéris (1993, 1998, 2005, 2007a, etc.).

L'élaboration du corpus d'étude est effectuée en deux étapes: la pré-enquête et l'enquête, comme le suggère J.-L. Calvet (2004, 2007). Dans la pré-enquête, nous avons adopté un questionnaire ouvert, composé de questions d'identification de l'interlocuteur et d'autres questions d'opinion ciblant la mise en mots écrits de ce qu'on penserait des raisons du recours systématique aux désignations parallèles des rues dans la ville de Tizi-Ouzou (Algérie). Six odonymes parallèles sont concernés par cette étude : la «Rue des douze salopards», désignée officiellement «Rue des Frères Belahdj», sachant que les Frères Belhadj sont des héros de la guerre de Libération nationale en Algérie) ; la «Rue de la paix», à laquelle a été attribuée la désignation officielle de «Rue Zidane Amar» (un autre martyr de la Révolution algérienne) ; la «Rue de l'hôpital», appelée officiellement «Rue Lamali Ahmed», ( un autre martyr de la guerre de Libération nationale); la «Rue du CEM sud», connue sous le nom officiel de «Rue des Frères Ouchen», (eux aussi sont des martyrs de la Révolution de Novembre 1954), et la «Grande rue», baptisée officiellement du nom de «Rue Abane Ramdane», (militant politique et révolutionnaire algérien ayant joué un rôle clé dans l'organisation de la lute indépendantiste en Algérie.

Vingt-cinq questionnaires ont été constitués et distribués lors de la préenquête. Les questionnaires adoptés dans la pré-enquête ne sont pas structurés, et l'ordre des idées ne conduit pas à un objectif quelconque précisé au préalable. Les questions formulées ont pour but juste le recueil des discours provoqués et coconstruits dans l'échange avec les partenaires de l'interaction au sujet de l'usage d'odonymes parallèles dans la ville de TiziOuzou. Les productions discursives écrites obtenues nous servent, ensuite, dans l'élaboration d'un guide d'entretien global que nous personnalisons selon les réponses apportées par les partenaires de l'échange retenus pour l'enquête.

Des prénoms fictifs ont été portés sur les questionnaires récupérés. Le repérage des auteurs des mots et le choix de ces derniers pour des entretiens durant lesquels nous soumettrons leurs propos à discussion, mais également de faire ressortir les éléments de catégorisation des odonymes parallèles dont on se sert dans la ville de Tizi-Ouzou en discours épilinguistiques, ont été faits à base du contenu et les mots utilisés dans les réponses aux questionnaires de la préenquête. C'est de cette manière donc que sont ressortis les éléments à base desquels est constitué le guide d'entretien global dont le but est de désambiguïser ces éléments de catégorisation des odonymes parallèles en discours verbal. Et à la lecture des réponses obtenues, certaines formulations semblent ambigües et nécessitent donc des discussions directes avec les enquêtés en vue de les désambiguïser, bien qu'elles apparaissent fonctionner comme des évidences et des vérités générales qui ne requièrent aucune explication pour leurs auteurs.

Ces ambigüités constituent pour nous le fil conducteur du guide des entretiens avec ceux parmi les interlocuteurs ayant accepté de prendre part à l'enquête autour des propos que chacun a mentionnés dans sa réponse au questionnaire de la pré-enquête. Entre autres mots et idées, dans les réponses mentionnées dans les questionnaires récupérés, qui doivent, à notre sens, être clarifiés avec plus de détails pour les désambiguïser, nous citons des qualificatifs et des jugements, dont la présence discursive se confirme dans les entretiens avec les mêmes enquêtés qui les formulent dans les réponses de la pré-enquête. Cela concerne surtout des segments discursifs formulés par les participants à l'enquête qui considèrent les désignations parallèles de rues à TiziOuzou comme étant soit maintenus en usage par dérision ou utilisées pour contrecarrer 
l'arabisation, ou bien une pratique sociale involontaire, ou encore un procédés de détournement de l'histoire, etc.

Dans la deuxième étape, nous sollicitons directement la parole auprès des personnes sélectionnées selon leur disponibilité et le profil retenu dans la préenquête afin de désambiguïser les mises en mots. L'enquête est conduite par nousmêmes (Enquêteur, désigné avec l'initiale E plus loin dans la partie Résultats et discussion), en français étant donné que celui-ci constitue notre langue de compte rendu. Le kabyle est sollicité quand même (par les partenaires de l'interaction notamment) tantôt "pour s'assurer d'avoir dit ce qu'on veut, [tantôt] pour riposter à un propos jugé mal interprété, pour ironiser, se moquer, etc.» (Sini, 2007, p.187). Il faut dire, d'autre part, que le recours, au cours des échanges verbaux, à la langue arabe est insignifiant. Seuls quelques termes arabes ont été énoncés par certains parmi les partenaires de l'interaction orale que nous présentons d'ailleurs. Les six participants aux discussions sont: Mahrez (M), Ramdane (désigné par l'initiale $\mathrm{R}$ dans la partie Résultats et discussion), Lila (mentionnée avec l'initiale $\mathrm{L}$ dans la partie Résultats et discussion), Boualem (cité avec l'initiale B dans la partie Résultats et discussion), Hafid (H) et Tassadit (citée avec l'initiale T dans la partie consacrée à l'analyse des mises en mots. Pour sa part, Ramdane (R) considère l'acte de nommer les rues par les noms de chouhada (désignant, en Algérie, les moujahids ou les morts au champ d'honneur pendant la guerre de Libération s'étalant de 1954 à 1962), s'inscrit dans cette démarche visant à réhabiliter l'histoire du pays. Il fait entendre que désigner une rue par une dénomination autre que celle consacrée officiellement peut avoir «une arrière pensée de faire oublier l'histoire et la culture du pays». Et pour Lila (L), c'est plutôt l'école qui n'a pas joué son rôle dans la vulgarisation du domaine de l'onomastique. C'est pourquoi, d'après elle, l'appellation parallèle des rues est largement généralisée dans le milieu urbain. Ainsi, selon l'interlocutrice, le français est utilisé dans la dénomination parallèle des rues pour contrecarrer l'arabisation. De son côté, Boualem (B) pense que l'usage de l'odonyme parallèle Grande rue n'est ni motivé par «un choix délibéré» ni produit «d'un consensus social conscient», mais plutôt «le résultat d'un héritage» difficile à situer ou à dater. Aux dires de Boualem, la dénomination parallèle des rues dans la ville de Tizi-Ouzou est l'œuvre de pratiques sociales inconscientes. Tandis que Hafid $(\mathrm{H})$ estime que le maintien du dénominatif parallèle Rue de l'hôpital à la place de la désignation officielle Rue Lamali Ahmed se justifie par le fait qu' «il est plus facile de voir une bâtisse de l'envergure de l'hôpital qu'une petite plaque qu'on ne sait pas où trouver». Tassadit (T), pour sa part, estime que le recours aux odonymes parallèles comme mode de désignation des rues dans la ville de Tizi-Ouzou participe largement à la déformation de la toponymie et à l'instauration de l'anarchie dénominative en milieu urbain. Et, le recours au français pour la formulation et la mise en circulation des odonymes parallèles dans la ville a, selon l'interlocutrice, lien au statut prestigieux qu'offre cette langue aux yeux des utilisateurs.

Nous nous servons d'un guide d'entretien conçu selon les résultats des analyses des données de la pré-enquête. Les échanges verbaux avec les interlocuteurs ont été conduits de telle sorte à provoquer la parole, à solliciter des clarifications en ce qui concerne des mots ou des tournures utilisés dans les réponses formulées par les participants à l'enquête. Ainsi, nous avons procédé de manière à permettre aux échanges verbaux de prendre l'allure de discussions familières. Ce qui permet la coconstruction dynamique du sens en langage. Et pour une meilleure libération de la parole, nous avons adopté, dans chacun des entretiens, la forme (ou les formes) de langue, c'est-à-dire le/les langage(s) des interlocuteurs. C'est une démarche axée sur l'enregistrement des paroles des enquêtés directement au moment de leur (re)production dans des situations d'interaction, en ciblant l'observation de la réalité telle qu'elle est. C'est, d'après Blanchet (1998), une «(...) démarche d'élaboration d'un savoir au service des finalités multiples 
qui s'insèrent dans un projet global de l'homme pour décrire, comprendre son environnement et les événements qui $\mathrm{y}$ déroulent» (p.17).

Nous avons été amenés à l'élaboration d'un guide d'entretien global reliant chacun des praxèmes et des productions praxématiques qui redondent dans les réponses aux questionnaires de la préenquête. Précisons que même avec le même regard de catégorisation, souvent les logiques dans les questionnaires changent dans les interviews. Ce qui constitue d'ailleurs des limites que montre la conduite des échanges verbaux. C'est aussi ce qui rend plus grande notre responsabilité quant aux productions discursives co-construites au moyen du guide d'entretien élaboré à la lumière des données fournies par la préenquête pour mener la collecte des paroles co-construites.

Le verbal est un aspect des échanges provoqués, co-construits et enregistrés. Nous avons tenu compte de la gestuelle, de l'intonation, des ruptures de programmes, des bégaiements et répétitions, des prolongements vocaliques..., car selon l'approche praxématique, ces éléments contribuent à la co-construction du sens des discours tenus sur les odonymes parallèles dans la ville de Tizi-Ouzou. Ces éléments sont donc analysables. La saisie des entretiens est limitée à l'aspect verbal des échanges, tout en notant les éléments paraverbaux qui nous semblent, au moment de la transcription écrite, participer à la fois au déroulement de l'interaction mais aussi à la construction du sens.

Le travail de l'analyse commence d'ailleurs à ce niveau, et ce, surtout en ce qui concerne le choix de mentionner tel ou tel élément pour faire ressortir de la matérialité verbale des mises en mots co-construites avec les enquêtés le sens en action autour des représentations faites en discours épilinguistiques à propos des odonymes parallèles dans la ville de Tizi-Ouzou. Les entretiens ont été saisis au moyen de l'écriture en français lorsqu'il est question des échanges en cette langue. La même langue a été également adoptée à la fois pour transcrire les quelques propos tenus en kabyle et les rares mots énoncés en langue arabe et pour mettre en avant la lisibilité du message. Aussi, étant donné que le français est la langue de notre compte rendu, la version française des propos tenus en kabyle est donnée entre crochets dans les chapitres consacrés à l'analyse des mises en mots.

Le corpus verbal n'est exploitable qu'après transcription. Des écoutes successives s'imposent car les pauses, les hésitations etc., marquées pendant l'échange, sont considérées, en praxématique, comme des données importantes dont il faut tenir compte pendant la transcription sur papier, d'autant plus qu'elles servent de repères dans la compréhension et la mise en mots des dires. L'unité de base dans notre corpus est le tour de parole. Et chaque tour de parole est suivi des initiales de l'interviewé ou de l'enquêteur (que nous sommes) et du numéro de l'intervention. Il nous était difficile d'évaluer de la part exacte qui revient à chacun des auteurs de ces coénonciations. Nous rendons compte toutefois de l'importance du fait que c'est nous-mêmes qui avons effectué les enregistrement des mise en mots constituant le corpus à la base de ce travail au lieu de les avoir fait faire par d'autres personnes. Cela aurait garanti une certaine neutralité et objectivité dans les analyses conduites et réduit les risques d'implication de notre subjectivité dans les paroles provoquées et recueillies.

\section{RÉSULTATS ET DISCUSSION}

Ce qui est analysé ici ce sont donc les représentations sociales que l'on se fait d'odonymes parallèles dans les discours épilinguistiques sur le plan de leurs élaborations linguistiques dans le cadre des discussions coproduites auxquelles nous avons participé. Ces dernières constituent une co-construction impliquant des tensions dans le réglage du sens dans les productions discursives produites dans des conditions particulières de co-construction en rapport avec le contexte social, politique et culturel dans lequel nous évoluons, les enquêtés et nous-mêmes.

Le travail porte sur la trace de l'activité épilinguistique des participants aux échanges 
verbaux pour rendre compte du processus conversationnel et dynamique de la coconstruction du sens dans les paroles tenues au sujet des odonymes parallèles dans la ville de Tizi-Ouzou. Ce sens est saisi dans cette perspective que forment au même temps la représentation et les représentations qui découlent des marques dialogiques et dialogales des productions praxématiques provoquées et déroulées sous une forme conflictuelle, dynamique et momentanément accomplie.

Quels sont à présent les procédés linguistiques à la base de la construction de ces représentations que se font ces interlocuteurs des odonymes parallèles dans la ville de Tizi- Ouzou en discours ? Des paroles provoqués et recueillies dans des conditions de l'intersubjectivité qui nous a liés à nos interlocuteurs lors de l'enquête, il ressort que les discours épilinguistiques y sont construits selon un mode qui dépeint les odonymes parallèles d'éléments constituant un enjeu idéologique ou encore d'éléments par lesquels la valeur morale des combattants de la Révolution est mise en péril. Il ressort à titre d'indication du discours co-construit avec Ramdane que la dénomination des rues en particulier, et des espaces publics de manière générale, n'a pas subi de changements de procédure depuis longtemps. En effet, pour lui, l'odonymie n'a pas du tout pour réputation de puiser dans le creuset de la culture par exemple. Les chouhada s'empare de la reconnaissance officielle et éternelle de la nation, «au détriment» des hommes de culture et des scientifiques qui, eux, demeurent ignorés, marginalisés. Ils n'ont pas le droit de cité dans le paysage odonymique. Cet état de fait ne serait-il pas à l'origine de l'usage d'odonymes parallèles ? Car les appellatifs parallèles sont mis en circulation dans la société alors que les dénominations consacrées officiellement sont contrecarrées. Les praxèmes offensifs actualisés dans les mises en discours co-construites avec Ramdane n'ont-ils pas, justement, pour souci énonciatif de rendre compte de cette valorisation du legs mémoriel des chouhada au détriment des hommes ayant rendu service à l'humanité autrement, en se consacrant pour la culture, de la science, l'art...? C'est la suite de l'étude des paroles coproduites avec ce partenaire de l'interaction orale qui nous donnera des éclaircissements à ce propos.

En effet, pour Ramdane, le remplacement systématique de la dénomination officielle Rue des frères Belhadj par l'odonyme parallèle Rue des douze salopards, à titre d'illustration, n'est ni un usage innocent ni dénué de sens. Il s'agit ici d'un «emploi péjoratif» ( $\mathrm{R}$ 2) qui n'est rien de plus qu'un usage faisant allusion ironiquement «aux acquéreurs» (R 2) installés tout au long de cet important boulevard traversant (la Nouvelle-ville) de Tizi-Ouzou et autour duquel s'articule une vie économique intense.

Nous saisissons tout de suite l'énoncé «genre d'acquéreurs» qui nous semblait floue. La désambigüisation de l'expression, la nature de ces acquéreurs par exemple..., a été vraiment difficile pour Ramdane. Le réglage de sens de cette formule n'était pas si facile à faire émerger dans l'échange verbal. Les premières tentatives de désambigüisation des praxèmes actualisés pendant les premiers tours de paroles avec Ramdane, où sont évoqués ces acquéreurs, se retrouvent face à d'autres programmes de sens orientant l'échange verbal dans une autre voie de discussion. Il y avait comme une espèce de volonté de changement de discussion affichée chez notre interlocuteur dès qu'il prononce le praxème «acquéreur». $C^{\prime}$ est là que nous retenons donc un praxème qui cache un enjeu et dont le réglage de sens semble complexe. L'orientation que tente d'accorder Ramdane au fil du discours est teintée de doute que démasque l'emploi répétitif du praxème «peut-être» (R 2), comme le confirme cet extrait du début de l'entretien avec Ramdane:

E1/ pourquoi on recourt souvent à la dénomination parallèle rue des douze salopards pour désigner la rue des frères belhadj ?/

R2/ c'est c'est l'usage/c'est l'usage euh qui certainement était justifiéldans l'esprit des gens qui l'ont appelé boulevard des douze salopards/certainement que ça faisait allusion euh péjorativement à : aux acquéreurs qui étaient peut-être connus pour leur mal euh 
pour leur euh euh pour leur situation euh disons euh socio-financièrelqui étaient peut être mal vus par la population/ils l'ont donc appelé boulevards des douze salopards faisant référence euh aux comportements de ces genslàl

S'agit-il ici d'une incertitude qui cache un à-dire difficile à extérioriser ? Sachant que l'instance de l'à-dire désigne l'activité inconsciente qui concerne la programmation praxématique, phrastique, textuelle, etc., précédant l'élaboration concrète des unités linguistiques en inconscience. N'y a-t-il pas un programme de sens en gestation chez notre partenaire de l'interaction qui véhicule des paroles désambigüisantes pouvant mener à la l'explication du fait de toucher justement à la réputation des Frères Belhadj? La réponse que donnerait-il à notre parole curieuse serait-elle affirmative? Nous ne perdons pas en tout cas de vue l'expression actualisée par Ramdane qui garde quand même la retenue pour un bon moment, en multipliant les hésitations, marquées par «euh» $(\mathrm{R} 2)$, juste après l'actualisation du praxème «acquéreurs», toujours dans le même tour de paroles.

Notre parole curieuse (en E 3), sollicitant plus de détailles à ce propos, libère partiellement la pulsion communicative de notre interlocuteur. En mobilisant une série de qualificatifs, Ramdane sort peu à peu de sa réserve pour soutenir que les Frères Belhadj font partie d'une catégorie d'hommes «intègres» de «valeureux combattants» et «sérieux» qui ont sacrifié leur vie et donné leur sang pour libérer le pays. Ramdane finit par libérer partiellement sa pulsion communicative et dire, en M 8, que ces révolutionnaires (les frères Belhadj) sont outragés par ce genre de dénominatif parallèle, largement adopté et circulé dans le milieu urbain. Mais nous remarquons ici que le partenaire de l'échange verbal tente de réorienter encore une fois le discours dans un autre sens. Il range de côté le praxème «acquéreurs» et met face à face les énoncés «douze salopards» et «frères Belhadj». Il ne désambigüise jusque là pas le praxème constituant l'enjeu principal de l'interaction langagière engagée jusque-là. Il use de camouflage comme stratégie discursive, en soumettant à la discussion deux énoncés qui s'opposent.

A ce moment là, nous reformulons la question pour éviter justement une orientation autre à l'interaction et recentrer l'échange verbal en mettant en avant la réputation des frères Belhadj. Ramdane ne manifestait pas de gêne pour assumer explicitement que l'image de marque de ces combattants se trouve souillée par cette appellation dénigrante (rue des douze salopards) qui, en revanche, revivifie, dans l'imaginaire populaire, l'image d'acquéreurs ayant spolié de manière «mafieuse» (R 8) des lots de terrains sis sur la rue dénommée officiellement, et contre toute attente, de leur nom. C'est ici-même donc que Ramdane libère définitivement sa pulsion communicative après une hésitation et déclare en effet la nature «mafieuse» des acquéreurs dont il avait parlés tout au début de l'échange verbal :

E3/ mais est-ce que justement euh ça ne touche pas la réputation des frères belhadj ?/

$\mathrm{R} 4 /$ euh ouilen réalité ça jette de l'opprobre sur euh les frère belhadj qui eux ont une renommée hautement plus HONNETE/ hautement plus intègrel et euh qui fait référence à la grande valeur des chouhada de notre pays/ euh dans la guerre de libération nationalel semmeh iyi [excusez-moi]// l'usage agi[cet usage] qui est fait euh de cette déformation dans des situations EXTREMES euh à un nom de gens intègres honnêtes pour passer à un nom aussi malfrat app des douze salopards euh/

Donc ici, les paroles de notre interlocuteur désambigüisent le praxème représentant un enjeu dans l'échange et dénoncent, quelque part, la mise en péril de la réputation des frères combattants de la Révolution. Le résultat : on continue à présent à s'orienter dans ville en recourant à une dénomination parallèle de la rue dont la porté sémantique symbolise plutôt des acquéreurs ayant bénéficié illégalement de lots de terrain. L'odonyme officiel remémorant les chouhada reste par contre toujours méconnu.

Pour Ramdane, le recours au nom parallèle Rue des douze salopards participe à la déformation comme il décline péjorativement les combattants de la 
Révolution. Une «image de pureté» (R 26) se retrouve salie par des...«antirévolutionnaires» (R 26). Parler de salopards devrait pourtant, selon les mises en discours de l'interlocuteur, concerner plutôt les "prédateurs des biens $d u$ pays» ( $\mathrm{R}$ 26) dont on se sert du nom pour désigner parallèlement une rue dans la ville de Tizi-Ouzou. Selon le raisonnement de Ramdane, user du nom douze salopards en guise de dénomination parallèle d'une rue dans cette ville ne peut avoir d'autres interprétations si ce n'est celle signifiant le contrepoids qui contrecarre la valeur morale des Frères Belhadj.

Sur un autre plan, il nous semble «peu objectif» de ne pas aborder ici la place du français dans la circulations des désignations parallèles de rues à Tizi-Ouzou sans l'interroger à partir de notre rapport avec les enquêtés et de notre environnement respectif qui font que les décodages que nous avons proposés de ces discours impliquent bien sûr des attitudes mettant en avant notre statut d'universitaire en quête de l'objectivité mais encore de citoyen évoluant dans le milieu à partir duquel nous nous proposons d'expliquer les mécanismes linguistiques de formation des représentations que ces partenaires de l'échange se font des odonymes parallèles à l'instant même des échanges verbaux.

Ce n'est pas par aliénation que la population tizi-ouzienne recoure à la langue française pour formuler les noms par lesquels l'on indique parallèlement les rues dans la ville (L 20). Pour les tizi-ouziens, l'usage de cette langue est stratégique. Le français, pour eux, est adoptée en signe de résistance à la l'arabe imposé par l'Etat. Une langue qui n'est pas dans l'usage et qui est rejetée. «L'arabe n'est pas dans l'usage» (L 20) à Tizi-Ouzou. Pour Lila, on recourt communément à la langue française «même si elle est (...) est un peu écorchée [et] complètement (...) malaxée» (L 20) :

L 20/je pense que la langue française euh à tizi-ouzou ce n'est pas parce que les tiziouziens ou les gens de la kabylie sont encore aliénés/ loin de làl c'est un choix aussi stratégiquel et par euh on a voulu ignorer la langue euh autochtone la langue qui est un repère identitaire aussil à partir du moment où on l'a euh REjettée pour lui SUbstituer une langue qui n'est pas dans l'usagel il faut le direl l'arabe n'est pas dans l'usagel donc on lui a substitué cette langue et du coup les tizi-ouziens REjettent la première c'est-à-dire la langue euh arabe pour euh opter pour la langue euh françaisel et euh elle est/ nous remarquons qu'aujourd'hui la langue française euh est VRAIment dans l'usage même si elle est euh elle est un peu écorchée elle est euh elle est complètement euh malaxéel

E 21/mise dans un moule typiquement kabyle/ c'est çal

L 22/oui : on l'a mise dans un moule typiquement kabyle/ mais on CONtinue à la parler quand mêmel c'est euh on l'a coulél moi j'ai toujours dit ce sont des phrases qui sont KAbyles sur le plan structurel sur le plan/mais on emprunte un lexique français/ et c'est ce qui se passe maintenant/ donc c'est un choix beaucoup plus euh qui marque une euh une identitél donc on parle en français pour dire que nous ne sommes pas arabes/euh ça peut paraitre paradoxal mais c'est le cas/ donc on parle kabyle français et non pas kabyle-arabe/voilàl

Au début du tour de paroles L 20, nous remarquons l'émergence $d u$ « je» dans les paroles tenues par Lila. Et théoriquement, lorsqu'un sujet parlant émet un discours, il déclare par cet acte à la fois son existence et son appartenance à un espace social. C'est le cas de cette interlocutrice dans cette séquence énonciative où elle se déclare faisant partie du monde, comme elle apporte de la réalité à son propos par le «je». Selon F. Madray et R. Lafont (1976), ce «je» constitue un indice de présence du sujet parlant, le critère de base de l'existence du monde «et que le langage est ancré, comme locution, dans cette existence» (p. 90). Ainsi, à partir de ce «je» qui marque la présence du sujet parlant au monde, l'Ici et l'Ailleurs de la parole se dessinent comme se déterminent les fréquences temporelles, envisagées en «ascendance» ou en «descendance»; deux notions qu'emprunte à la linguistique guillaumienne la linguistique de la parole, où le temps ascendant "correspond à un flux temporel qui tend vers l'avenir du sujet, et le temps descendant à un flux qui tend vers son passé» (Ait Sahlia-Benaissa, 1999, p. 31). En effet, d'après les dires de Lila, «on a voulu ignorer la langue (...) autochtone» (L 20), c'est-à-dire le kabyle, en diffusant massivement et contre toute attente et toute 
volonté la langue arabe. En signe de résistance, les tizi-ouziens adoptent la langue française pour contrecarrer la politique d'arabisation.

Aussi, il ressort des mêmes discours que le français, à l'aide de laquelle ces attributs parallèles par lesquels les rues sont désignés à Tizi-Ouzou sont formulés et mis en circulation, constitue un moyen linguistique controversé, dans la mesure où cette langue est utilisée ainsi pour exhiber un statut social prestigieux ou bien comme langue de consensus. S'agissant du recours au français lors de la dénomination parallèle des rues dans la ville de Tizi-Ouzou, l'interlocuteur dit que celle-ci est la langue $\mathrm{du}$ consentement dans la communication quotidienne. Selon les paroles de Boualem, le recours à cette langue qui marque ainsi largement le paysage odonymique de la ville «peut être l'une des bases du poids de la mémoire» (B 18). La question du code linguistique utilisé pour désigner parallèlement les rues n'échappe pas, pour lui, aussi au poids de la mémoire et d'un certain nombre de traditions complexes. Car dans la mémoire populaire de la région particulièrement, il y a toujours tendance à formuler en français les noms par lesquels on désigne les espaces dans le milieu urbain.

Il y a une survivance de cette tendance à désigner les rues..., «parce que les objets de la vie quotidienne quand ils sont désignés dans une langue étrangère, comme le français, ils assurent plus de consensus que quand ils sont désignés dans des langues autres» (B18). Un peu plou loin, dans la discussion, notre interlocuteur donne une autre interprétation nouvelle à l'usage du français qu'on utilise pour la mise en circulation des odonymes parallèles dans la ville de Tizi-Ouzou. Nous réitérons en E 17 la même question à propos de l'usage du français et le participant à l'enquête de dire que la population s'est habituée à indiquer parallèlement les rues en français parce que depuis l'époque coloniale, et même après l'indépendance, le français était la langue de l'administration. Quelques tours de paroles un peu plus loin, il en rajoute une autre interprétation : pour lui, la Rue des frères Ouchen est un odonyme parallèle formulé et largement circulé en français «parce que nous sommes dans une région kabylophone assez fronDEUSE par rapport à la langue arabe» (Boualem 38).

Dans ce tour de parole, Boualem actualise un praxème plutôt révélateur : «fronDEUSE» qui entraine un réglage de sens tel que celui donné à l'usage du français comme moyen de mise en circulation des odonymes parallèles lors de l'échange avec Lila dans le chapitre précédent. Boualem catégorise l'usage du français par rapport à la langue arabe.

Mêlant récit et commentaire, Tassadit témoigne, dans l'échange, des rapports de force entre francisants et arabisants pendant la période de sa scolarisation au début des années 1950 ; pour elle, la controverse que provoque l'usage du français dan le milieu tizi-ouzien s'exprime sous forme de lutte:

E43/et à l'école? dans la cours ?/

$\mathrm{T} 44 /$ à l'école c'était la langue françaisel dans la cours dans la salle de classe alors et pourtant les les enseignants ne nous interdisaient pas de parler autre chose en dehors de la classel chacun pouvait parler ce qu'il parlait/ c'était jamais un problème de languel mais dans la salle de classe la langue euh d'enseignement la langue de communication c'était la langue françaisel et rien d'autrel et c'est bien que même dans la cour de l'école vous entendrait rarement rarement quelqu'un quelques copines qui parlaient le dialectal de tizil l'arabe dialectal d'icil et le kabyle rien du tout/

E45/ donc euh on a hérité ici à tizi-ouzou de : cette coutume-làl

T46/ il y a comme une espèce de coutumel on sait pas comment la placer/ mais on se sente plus à l'aise plus valorisé en quelque sorte de parler français/ ça veut dire vous êtes éduqués vous êtes instruits voilà surtout ça vous êtes instruits au même titre que tous les français que tous les gens/ donc tous le monde c'était mis au français/ parce que qui ne parle que le kabyle entièrement ça veut dire analphabète et ignorant/ c'est : c'est carrément çal

E47/c'est ça la distinction?/

T48/la distinction c'était çal quelqu'un qui ne parlait pas français ça veut dire qu'il n'a jamais mis les pieds dans une salle de classe et un petit peu euh comment dirais-je ? un peu dévalorisé en quelque sorte aux yeux de la sociétél quitte à baragouiner la langue française les gens se mettent à la langue françaisel chez le commerçant d'à côté contrairement à maintenant je fais mes 
commissions entièrement en kabyle/il y a 55 ans je faisais mes courses en parlant en langue française à un kabyle comme moil mais vraiment !/ c'était comme une euh une espèce de complexel ou comment le placer ça? je ne sais pas mais euh c'était tout-à-fait naturel/ c'était naturellement qu'on utilisait la langue française uniquement/ pour la langue française il y avait une emprunte telle que euh même les noms sont un petit peu dénaturés/ dans la cour on parlait français/ un petit peu l'arabe dialectall un petit peu avec les filles de la haute ville/ très peu/ c'est bien après il $y$ avait même une scission entre les groups/il y avait les groupes des filles de la haute ville et nous les citadines qui venons d'ailleurs beaucoup plus proches des françaises/

Cette (longue) séquence discursive présente en faite une réflexion sur la situation sociolinguistique en rapport avec l'usage du français et de l'arabe (et à un degré moindre le kabyle) dans la ville de Tizi-Ouzou, dont la langue française constituait (et continue à présent de constituer) l'enjeu principale dans la gestion des langues avant et après l'indépendance de l'Algérie. Le positionnement du français, comparé aux autres langues en usage dans ce pays, est controversé. En effet, l'arabe continue de tirer sa force du soutien de l'Etat qui lui octroie des budgets et moyens; une langue que l'Etat algérien s'efforce d'imposer depuis le recouvrement de l'indépendance. Le français, en revanche, apparait pour d'aucuns parmi les masses populaires, berbérophones surtout, comme la langue d'une incontournable modernité et de prestige, même si reléguée officiellement au rang de langue étrangère. C'est cette forme de catégorisation d'ailleurs que confère Tassadit à cette langue dans ses paroles (T44, T46, T48). Par ailleurs, selon les mises en discours de la même enquêtée, l'adoption de la langue française tant dans la formulation que dans la mise en circulation des odonymes parallèles au sein de la ville de Tizi-Ouzou revêt un encrage social profond. Il n'était pas question, aux dires de Tassadit, de ne pas maîtriser cette langue car elle était une langue de prestige qui procurait un statut particulier à celui qui la maniait.

\section{CONCLUSION}

Afin de parvenir à dégager les mécanismes linguistiques à base desquels s'élaborent les représentations linguistiques que l'on se fait dans les productions discursives ayant lien au recours aux odonymes parallèles à la place des désignations officielles pour nommer les rues à Tizi-Ouzou, nous avons adopté les principes de la théorie praxématique associés à l'onomastique. La relation du langage au réel est au cœur de la problématique abordée dans ce travail qui vise aussi à saisir, dans les productions langagières, les marques distinctives des catégorisations du réel que véhiculent les désignations parallèles de rues dans les mises en mots que nous avons recueillies en interaction intersubjective avec les participants aux discussions ayant mené à la construction du corpus étudié.

Ce sont, en effet, les élaborations linguistiques des représentations sociales faites des appellatifs utilisés parallèlement à ceux consacrés officiellement en discours épilinguistiques qui ont été saisies et analysées dans ce cas d'étude. Elles forment une co-construction incluant des tensions dans le réglage $d u$ sens dans les constructions langagières constituées dans des situations particulières de coconstruction en rapport notamment avec le contexte social, politique et culturel dans lequel évoluent à autant les partenaires des échanges verbaux que nous qui avions conduit l'enquête sociolinguistique.

Aussi, l'hétérogénéité énonciative faisant écho aux discours en circulation dans la société dans laquelle évoluent ces interlocuteurs constitue la réponse affirmative à l'hypothèse méthodologique à la base de cette contribution. En plus, les partenaires de l'échange verbal font souvent preuve d'hésitation comme ils se trouvent, $\mathrm{d}$ 'autre part, en manque de parole requise pour la désambigüisation. Par moment, pendant les échanges verbaux, les enquêtés manifestent plutôt une certaine aisance à la fois dans le lien dialogal avec nous et dialogique avec les voix qu'ils invitent pour par exemple ironiser, insister sur un point précis ou faire part d'une tension intérieure, etc. 


\section{REMERCIEMENTS}

Je tiens à remercier vivement Pr SINI Chérif qui est pour moi un maître compréhensif, humain et disponible. Ses conseils, sa rigueur intellectuelle et ses critiques sont d'un apport sans égal à ma formation.

\section{RÉFÉRENCES}

Ait Sahlia Benaïssa, A. (1999). La construction de l'identité dans le langage : variations autour de l'identité algérienne (Thèse de doctorat inédite). Université de Rouen.

Atoui, B. et Benramdane, F. (2005). Mondialisation et normalisation des toponymes et des écritures : le cas de l'Algérie. Dans B. Atoui et F. Benramdane (dir.), Nomination et dénomination. Des noms de lieux, de tribus et de personnes en Algérie (p.187197). Oran : Crasc.

Barberis, J.-M. et Madray, F. (1986). Ratage d'actualisation et évitement des temps et des personnes en production discursive orale. Dans F. Madray (dir.), Praxématique et psychomécanique du langage (p.3762). Montpelier : Presses universitaires de la Méditerranée.

Barberis, J.-M. (1993). Sujet et espace imaginaire. Dans F. Madray et P. Siblot (dir.), Langage et praxis (p.274284). Montpellier: Université Paul Valéry.

Barberis, J.-M. (1998). Représenter l'espace de la ville en contexte interculturel: l'impasse' identitaire. Dans B. Maurer et P.-Y. Raccah (dir.), Linguistique et représentation (s) (p.3968). Montpellier : Pulm.

Barberis, J.-M. (2005). La ville et ses composantes: l'émergence des catégories en interaction orale. Dans L. Dubois et T. Bulot (dir.), Signalétiques et signalisations langagières des espaces de villes (p.3160). Moncton : Revue de l'université de Moncton.

Barberis, J.-M. (2007a). Quartier des uns, quartier des autres. La construction de l'espace urbain dans la parole.
Dans P. Bacot et S. R. Giraud (dir.), Mots de l'espace et conflictualité sociale (p.187-197). Paris : L'Harmattan.

Barberis, J.-M. (2007b). Les codes de la ville. Culture, langues et formes d'expressions urbaines. Dans J.-M. Barberis (dir.), Nommer la rue en interaction orale: conflit sur les mots et conflits sur le sens (p.77-110). Paris: L'Harmattan.

Bres, J. (2006). Encore un peu, et l'imparfait était un mode...L'imparfait et la valeur modale de contrefactualité. Dans A. Patard et J. Bres (dir.), Aspectualité, temporalité, modalité (p.149-176). Montpellier : Pulm.

Blanchet, A. (1998). Les techniques d'enquête en sciences sociales. Paris : Dunot.

Calvet, J.-L. (2004). Essai de linguistique. La langue est-elle une invention de linguistiques? Paris : Plon.

Calvet, J.-L. (2007). Pour une linguistique du désordre et de la complexité. Carnets d'ateliers de sociolinguistique, (1), 3-70. Repéré à https://www.upicardie.fr/LESCLaP/IMG/pdf/Calvet _CAS_no1_cle8e8652.pdf

Guillaume, G. (1998). Psycho-systématique du langage: principes, méthodes et applications. Paris : Klincksieck.

Lafont, R. et Madray, F. (1976). Introduction à l'analyse textuelle. Montpellier: Université Paul Valéry III.

Madray, F. (1984). Un exemple de communication oraculaire : l'épître à Philémon. Cahiers de praxématique, (2), 2-14. Repéré à http://praxématique.revue.org/3553.

Madray, F. (1989). Lecture de: Bernard Gardin, Langage et travail, études sociolinguistiques de discours ouvriers en entreprise. Dans P. Siblot (dir.), Réglage et dérèglement du sens (p.112-116). Montpellier : Pulm.

Siblot, P. (1986). Jacqueline Picoche : structures sémantiques du lexique français. Dans F. Madray (dir.), Praxématique et psychoméchanique du langage (p.73-89). Paris : Nathan.

Siblot, P. (1988). Sémiotique et praxématique: L'analyse du entre 
idéalisme et matérialisme. Dans P. Siblot (dir.), Introductions théoriques, interrogations épistémologique (p.7389). Montpelier : Pulm.

Sini, Ch. (2007). Contribution à l'analyse des raisons $d u$ choix d'une graphie pour écrire le Kabyle (Thèse de doctorat inédite). Université de Rouen.

Timzouert, D. (2015). Analyse praxématique de discours épilinguistiques autour d'odonymes parallèles dans la ville de TiziOuzou (Mémoire de magistère inédit). Université de Tizi-Ouzou. 